\title{
Interconnected high-dimensional landscapes of epithelial-mesenchymal plasticity and stemness
}

\author{
Running title: Semi-independent control of EMP and stemness
}

\author{
Sarthak Sahoo ${ }^{1,2}$, Atchuta Srinivas Duddu ${ }^{1}$, Adrian Biddle ${ }^{3,{ }^{*}}$, Mohit Kumar Jolly ${ }^{1,{ }^{*}}$ \\ ${ }^{1}$ Centre for BioSystems Science and Engineering (BSSE), Indian Institute of Science, \\ Bangalore, 560012, India \\ ${ }^{2}$ UG Programme, Indian Institute of Science, Bangalore, 560012, India \\ ${ }^{3}$ Blizard Institute, Barts and The London School of Medicine and \\ Dentistry, Queen Mary University of London, UK \\ Email: a.biddle@qmul.ac.uk (AB), mkjolly@iisc.ac.in (MKJ)
}

\begin{abstract}
Establishing macrometastases at distant organs is a highly challenging process for cancer cells, with extremely high attrition rates. A very small percentage of disseminated cells have the ability to dynamically adapt to their changing micro-environments through reversibly switching to another phenotype, aiding metastasis. Such plasticity can be exhibited along one or more axes - epithelial-mesenchymal plasticity (EMP) and cancer stem cells (CSCs) being the two most studied, and often tacitly assumed to be synonymous. Here, we review the emerging concepts related to EMP and CSCs across multiple cancers. Both processes are multi-dimensional in nature; for instance, EMP can be defined on morphological, molecular and functional changes, which may or may not be synchronized. Similarly, self-renewal, multilineage potential, and anoikis and/or therapy resistance may not all occur simultaneously in CSCs. Thus, arriving at rigorous functional definitions for both EMP and CSCs is crucial. These processes are dynamic, reversible, and semi-independent in nature; cells traverse the inter-connected high-dimensional EMP and CSC landscapes in diverse paths, each of which may exhibit a distinct EMP-CSC coupling. Our proposed model offers a potential unifying framework for elucidating the coupled decision-making along these dimensions and highlights a key set of open questions to be answered.
\end{abstract}

\section{Keywords}

Epithelial-mesenchymal plasticity, stemness, landscape, phenotypic plasticity, cancer stem cells, metastasis 


\section{Introduction}

Cancer metastasis is the phenomenon in which secondary tumours develop at organs distant from the site of the primary tumour. It is a deadly event, accounting for more than $90 \%$ of cancer deaths [1]. A key hallmark of metastasis-initiating cells is their ability to adapt dynamically to their varying microenvironments, a trait known as phenotypic plasticity [2]. This plasticity enables them to circumvent various bottlenecks in the metastatic cascade and eventually colonize distant organs. It can exist along multiple inter-connected axes. Two of the most well-studied axes of cancer cell plasticity are epithelial-mesenchymal plasticity (EMP) and stemness [3]. These two axes have been shown to drive one another in in silico, in vitro and in vivo studies [4], but a unique mapping of states along the EMP axis and along the stemness axis has not been achieved. The heterogeneity in genetic and organ-specific backgrounds complicates this approach, as such relationships are likely to be context-dependent and lacking universality. Another factor which acts as a roadblock in developing a comprehensive understanding of these inter-connections is the lack of rigor and consistency in defining these multi-dimensional processes across different studies [5], i.e. what is necessary and sufficient to claim that cells have switched between epithelial and mesenchymal states or stem and non-stem states? Here, we review the emerging literature on complexity in defining EMP and stemness as individual axes of plasticity, the bidirectional interconnections investigated between these two phenomena, and eventually present a framework that can reconcile potentially conflicting observations.

\section{The multi-dimensional aspects of Epithelial-Mesenchymal Plasticity}

For successful dissemination of cancer cells from a primary tumour site and eventual colonization of a distant organ, cancer cells must exhibit invasive and migratory features. For carcinomas, such traits are attained via trans-differentiation of cancer cells from a relatively more epithelial to a more mesenchymal state with altered migratory, invasive, proliferative and polarity features through a process known as Epithelial-to-Mesenchymal Transition (EMT). Traditionally, EMT has been tacitly assumed to be an "all-or-none" process, but it is becoming increasingly clear that it is not a binary switch [6]. Both mathematical modelling studies and experimental observations have reported the existence of one or more hybrid epithelial/ mesenchymal (E/M) states between the two extremes of pure epithelial or pure mesenchymal phenotypes [7, 8]. The number of hybrid E/M states that exist between pure epithelial and pure mesenchymal has been a topic of debate with no general consensus [8]. The number of hybrid states can also vary depending on the cancer subtype. Of course, a large enough number of discrete states may be better approximated as a continuum [9]. Notwithstanding the difference in number of such states reported, a more important question is the functional relevance of these hybrid E/M states.

The term EMT has been tacitly assumed to denote a discrete and unidirectional process. With increasing recognition of hybrid E/M states, alongside the fact that cells can switch phenotype bidirectionally along this spectrum [10], the term Epithelial Mesenchymal Plasticity (EMP) has been introduced as a more accurate description of the process. An open question regarding the EMP spectrum is how to quantify the relative stability of different states - more extreme epithelial or mesenchymal ones vs. the hybrid E/M states [11]. In a system that allows for co-existence of multiple stable states (phenotypes), 'spontaneous' transitions among states are possible but the propensities of all possible transitions cannot be assumed to be the same [12, 13]. For instance, depending on genetic and/or epigenetic background, dose and/or duration of the induction signal, EMT and/or its reverse MET (Mesenchymal-to-Epithelial Transition) may be reversible or irreversible [14-17]. Thus, under some conditions, transitions in one or more direction may be prohibited. Moreover, cells may not necessarily follow the same 'path' when transitioning from $E$ to M and vice-versa in the EMP landscape, and consequently may or may not arrive at their initial state [18-21]; thus quantifying their transition dynamics is crucial. 
Defining the 'paths' of EMP requires a comprehensive understanding of necessary and sufficient conditions to claim that a transition has taken place. The EMT status of a cell is usually assessed using three different methods - molecular signatures, morphological features and functional traits - which may or may not be synchronized among one another; for instance, a cell may undergo "molecular EMT" but not necessarily a concurrent "morphological EMT" [22-24]. This lack of consistency in defining EMT percolates to poor characterization of hybrid E/M states as well. For instance, are both these categories of cells equally eligible to be called hybrid E/M: cells which have undergone "molecular EMT" but not "functional EMT", and cells which have undergone "morphological EMT" but not "molecular EMT"? To add to the combinatorial complexity of defining hybrid E/M state(s), each of these individual dimensions - molecular, functional, morphological comprises multiple cellular traits. For instance, do both of these categories of cells qualify as hybrid E/M: cells that have increased mesenchymal markers but unchanged epithelial markers, and cells that have decreased epithelial markers but unchanged mesenchymal markers? (Fig 1).

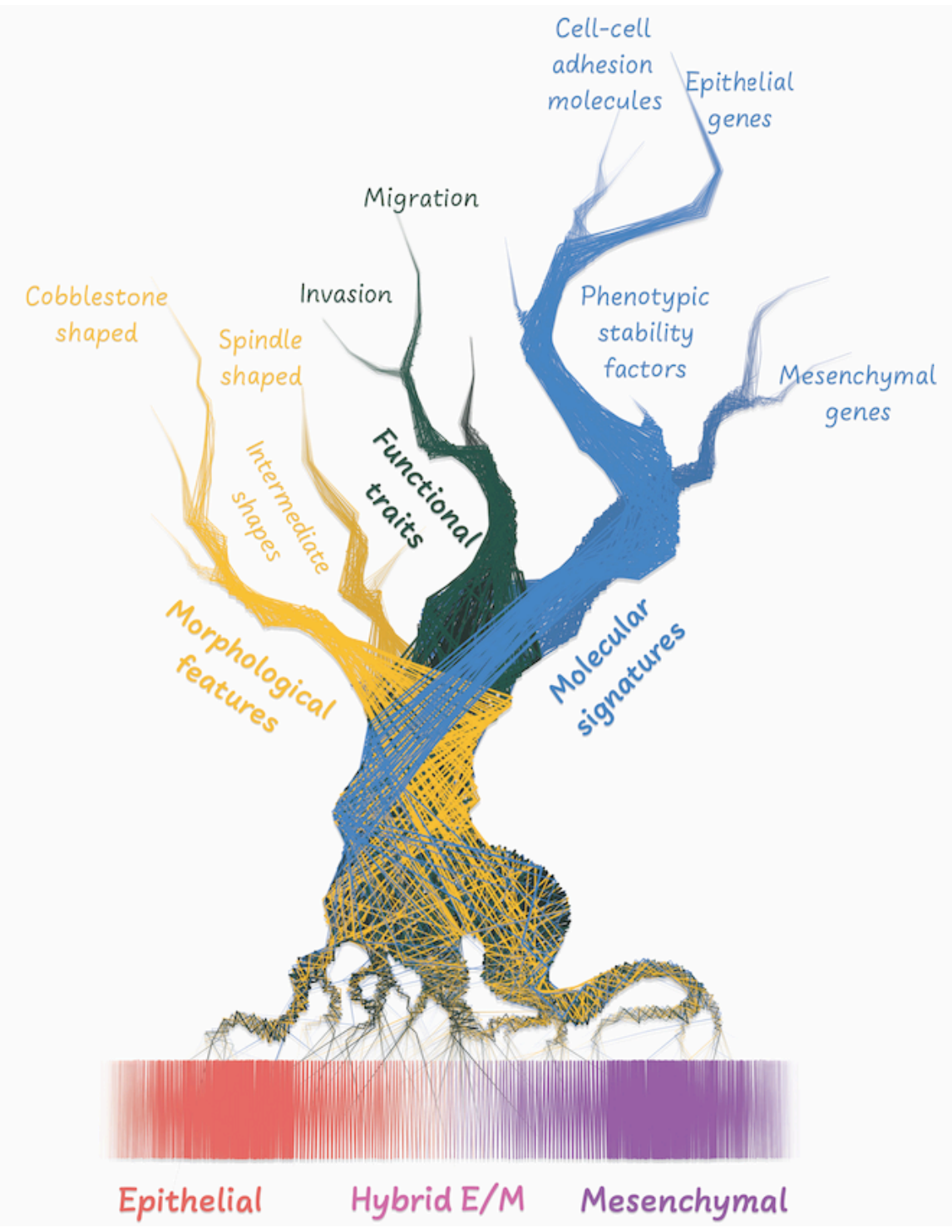

Figure 1: Multi-dimensional nature of EMP. The position of a cell along the EMP axis (shown below) is a composite score of its morphological, molecular and functional traits that are often expected to change as the cells traverse along this spectrum. The relative contribution of these different branches and their sub-branches to defining an "EMP score" remains to be identified. 
To quantify molecular changes associated with EMP, transcriptomic measures have been proposed that combine "epithelial" and "mesenchymal" scores, such as Singscore [25]. Only a few available metrics can score specifically for a hybrid E/M phenotypes, such as performing GSEA (Gene Set Enrichment Analysis) for a signature specifically associated with hybrid EMT phenotype [26] and/or assessment of collective cell migration [27, 28]. However, the accuracy of these existing metrics is currently unknown and they are unlikely to identify hybrid E/M phenotypes with sufficient resolution; robust pan-cancer hybrid E/M signatures are still lacking. Another possibility for identifying hybrid E/M status could be via the quantification of various 'phenotypic stability factors' in conjunction with the canonical epithelial and mesenchymal factors. For example, the mesenchymal factor SNAI2 has been shown to be robustly associated with a hybrid E/M phenotype [29-33] as is often expressed alongside CDH1 (E-cadherin), a well-known epithelial marker [34]. Relative levels of SNAI2 and CDH1 can thus stratify hybrid phenotypes along EMP spectrum [35].

On the other hand, cells can be classified into epithelial, mesenchymal or intermediate states by morphological features [36]. Epithelial cells are usually cobblestone shaped and often in close contact with their neighbours, while mesenchymal cells are more solitary and have distinctive spindle shaped morphology. Hybrid cells are relatively less well characterised, though have been seen to show slightly elongated phenotypes [37]. Functional assays such as scratch assays have also been used extensively to study the extent of EMT in cells [38], but no comprehensive comparison of functional attributes along the entire EMP spectrum yet exists.

\section{Stemness: What does it mean in the context of cancer?}

Stemness is the ability of a cell to self-replicate in order to perpetuate its own lineage, to give rise to more differentiated cell types to maintain a tissue, and to interact with its environment to maintain a balance between quiescence, proliferation, and regeneration [39]. Cells that exhibit such specific properties are called stem cells. Adult stem cells are observed to divide asymmetrically to produce progenitor cells that can, in turn, produce more differentiated cells that maintain tissue homeostasis in various organs [39]. A relatively recent development in quantifying the stemness of a cell has come from a global analysis of the transcriptome, thanks to the rapid advancements in high throughput sequencing of single cells [40, 41]. A stem cell with higher developmental potential is likely to exhibit a more diverse transcriptional profile than a differentiated counterpart [42].

Cancer stem cells (CSCs) can be viewed as malignant counterparts to adult stem cells. CSCs are generally considered to be a sub-population of the tumour mass that is capable of sustaining disease progression and driving post-therapeutic relapse. One of the widely used metrics to measure stemness in cancer is the ability of such cells to initiate a new tumour, also commonly known as the tumour initiation capacity (TIC). Interestingly, the origin of a primary tumour in many different cancer types has been attributed to the presence of CSCs that arise from either the tissue resident stem cells or differentiated cells that have undergone de-differentiation during transformation. CSCs are also observed to have various other associated attributes; however, some of these attributes are more likely to be associative in nature rather than an identification criteria per se. For example, CSCs are generally observed to be resistant to oxidative stresses and drugs and hence are likely to escape drug interventions and cause subsequent therapy relapse [43]. However, not all drug-resistant cells are necessarily CSCs. Similarly, CSCs have been associated with traits such as enhanced survival in suspension conditions (anoikis resistance) [44], ability to efflux Hoechst 33342 dye (side population) [45, 46], and enhanced migration [47] (Fig 2), but it is currently not at all clear whether these are hallmark features exclusive to CSCs. Further, a causative contribution of these traits in enabling cells to acquire and/or maintain stemness remains to be unequivocally established. 
The most common method used to identify CSCs is examination of various distinct cell surface markers. In many cases, the markers identified overlap with those used to identify either tissue resident stem cells or human embryonic stem cells. Cell surface makers such as CD44, CD133 and EpCAM have been identified as CSC markers in many carcinomas [48-50]. Intracellular markers such as SOX2, NANOG and OCT4 have also been implicated in maintenance of CSC fate in many cancer types $[51,52]$. Similarly, multiple signalling pathways have been shown to induce and/or maintain the CSC phenotype [53]. However, similar to EMP [54, 55], stemness is a dynamic trait which can be regulated in a non-cell-autonomous manner too. For instance, CSCs can secrete cytokines to convert their neighboring non-CSCs to CSCs $[56,57]$. It is important to note that these cell surface and/or intracellular markers, although strongly associated with a CSC phenotype, are not necessarily expressed equally in all CSCs. Conversely, the presence of one or more of these markers does not always guarantee a CSC phenotype and therefore other functional assays are required to establish stemness.

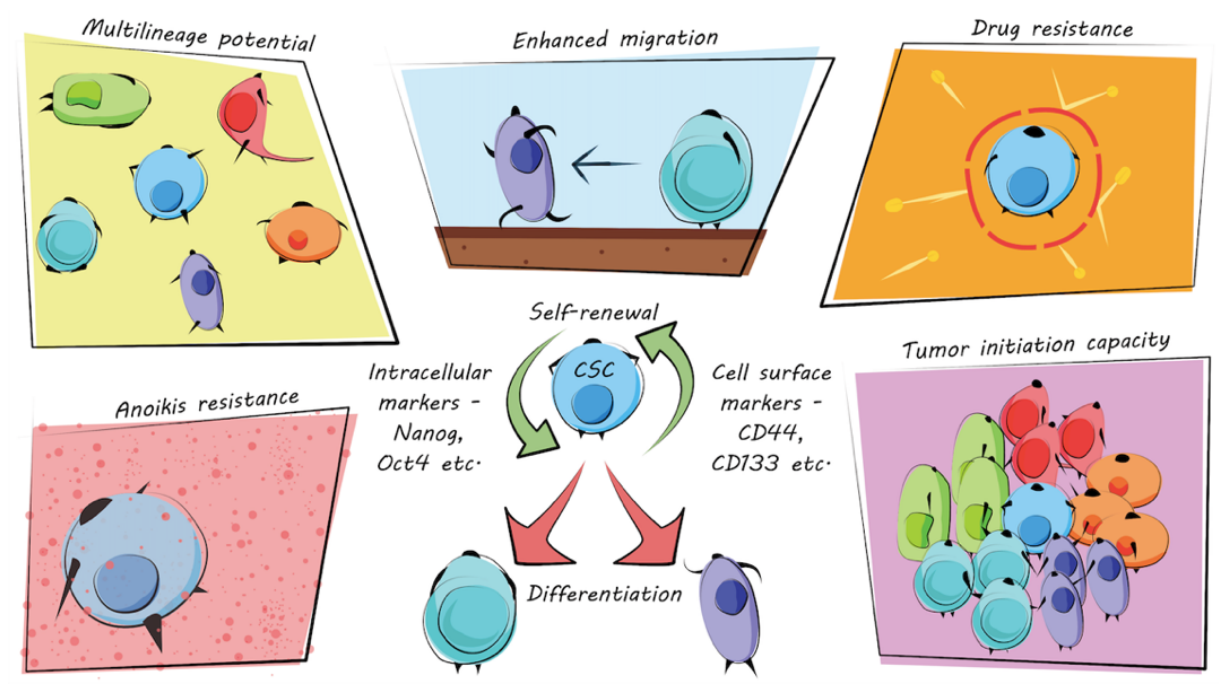

Figure 2: Multi-dimensional nature of CSCs. CSCs have been associated with various traits the most defining of which are the ability to self-renew and differentiation into other lineages. Other traits (anoikis resistance, drug resistance, enhanced migration) often co-occur alongside these defining traits, together with an enrichment of cancer-specific cell surface markers.

\section{Stemness on the Epithelial-Mesenchymal Plasticity spectrum}

The first set of observations connecting EMP and stemness were made in 2008, where a fraction of breast cancer cells induced to undergo EMT were shown to possess an elevated tumor-initiating capacity $[58,59]$. These observations were made when EMT was still seen as a binary process. They triggered further investigations between these two processes from a molecular perspective across cancer types, and the two axes were observed to be mechanistically inter-connected [60]. However, later studies which did not treat EMT as an 'all-or-none' process reported that hybrid E/M cells were equally or even more likely than mesenchymal cells to exhibit stemness [61-64]. Consistently, further investigation into CSC heterogeneity revealed that there may be subsets of CSCs - epithelial, mesenchymal and hybrid E/M (E-CSCs, M-CSCs, H-CSCs) [10, 50, 65]. Therefore, the emerging evidence points to EMT and stemness being semi-independent axes, i.e. not every cell undergoing EMT may acquire stemness and not every cell switching to be a CSC is mandated to show one or more features of EMT. Given the importance of both EMP and maintenance of stemness in the metastatic cascade, understanding the inter-connectivity between the EMP and stemness axes may help us shed light on key cellular processes driving metastasis. 


\section{Breast cancer}

Two seminal studies in 2008 demonstrated the association between EMT and CSCs, where a set of human mammary epithelial cells facing oncogenic activation or induced to undergo EMT showed an enriched CD44 ${ }^{\text {hi }}$ CD24 $4^{\text {lo }}$ population [58, 59], the claimed CSC subpopulation [66], as well as exhibiting increased tumor-formation ability as witnessed in vitro and in vivo. However, later investigations have indicated that epithelial cells with increased aldehyde dehydrogenase (ALDH1) activity are also capable of self-renewal and forming a tumor with cells of multiple lineages [67]. Immunofluorescence in primary human breast tumor tissues revealed that these two subsets of CSCs - CD $44^{\text {hi }} \mathrm{CD} 24^{\mathrm{lo}}$ and ALDH+ - are present at different spatial locations in a tumor. While the CD $44^{\text {hi }} \mathrm{CD} 24^{\text {lo }}$ cells localized at the tumor-invasive edge, the ALDH+ cells were located in the tumor interior [50]. Molecular profiling revealed that while CD44 ${ }^{\text {hi }} C D 24^{\text {lo }}$ cells displayed a mesenchymal phenotype, ALDH+ cells displayed a hybrid E/M phenotype [50,65]. These two subpopulations retained the ability to switch to one another as well as give rise to more differentiated cells, but whether this ability to spontaneously switch to another state is a cause and/or consequence of (partial) EMT/MET remains unclear. Moreover, whether this plasticity to switch back and forth between $\mathrm{CD} 44^{\mathrm{hi}} \mathrm{CD} 24^{\mathrm{lo}}$ and $\mathrm{ALDH}+$ phenotypes is necessary and/or sufficient to form tumors in vivo remains to be investigated.

Another set of investigations deciphered that while CSC enriched populations in luminal cell lines were enriched for a mesenchymal program, those in basal cell lines showed a more epithelial signature, suggesting an association of hybrid E/M phenotype with stemness in breast cancer [61]. When epithelial and mesenchymal subclones were established from HMLER cells, both these subpopulations seemed to fulfil only one of the two stemness conditions - while epithelial $\left(C D 44^{l o} C D 24^{\text {hi }}\right)$ cells had high plasticity, the mesenchymal $C D 44^{\text {hi }} C D 24^{\text {lo }}$ cells were more selfrenewing. Further analysis at a single-cell level identified a CD44 ${ }^{\text {hi }} C D 24^{\text {hi }}$ subpopulation which exhibited signatures of a hybrid E/M state, was enriched for ALDH+ cells and had approximately ten times more tumor-initiation potential than 'purely' epithelial or mesenchymal subpopulations (CD44 ${ }^{\text {hi }} C D 24^{\text {lo }}$, CD $44^{\text {lo }} C D 24^{\text {hi }}$ ) [61]. CD44 ${ }^{\text {hi }} C D 24^{\text {hi }}$ cells were seen to form tumors faster in vivo relative to $\mathrm{CD} 44^{\mathrm{hi}} \mathrm{CD} 24^{\mathrm{lo}}, \mathrm{CD} 44^{\mathrm{lo}} \mathrm{CD} 24^{\mathrm{hi}}$ and $\mathrm{CD} 44^{\mathrm{lo}} \mathrm{CD} 24^{\mathrm{lo}}$ cells, further establishing the aggressiveness of this hybrid E/M subpopulation [68]. Intermediate levels of ITGB4 (CD104) were reported to identify hybrid E/M breast cancer cells which also contained a majority of CSCs [69]. Co-culturing 'locked' epithelial and mesenchymal cells could not form as many tumors as 'plastic' hybrid E/M cells, pointing towards the possibility that plasticity, i.e. ability to traverse the EMP spectrum to varying degrees, contributes to stemness.

The association of hybrid EMT with stemness in breast cancer is increasingly reported. For instance, knockdown of cytokeratin-18 (CK18) in MCF7 cells induces a partial "molecular EMT" state (depleted E-cadherin, but upregulated EpCAM - epithelial cell adhesion molecule), and an increase in ability to form spheres and colonies [70]. Similarly, leader cells in collective cell invasion observed by $3 \mathrm{D}$ invasion assays are more likely to be CSCs and exhibit hybrid E/M phenotypes, showing co-expression of E-cadherin, N-cadherin and NANOG [71].

\section{Lung cancer}

CD133 is a canonical lung CSC cell surface marker. CD133+ lung cancer cells can self-renew as well as generate a progeny of differentiated cancer cells [72]. Treatment of various non-small cell lung cancer (NSCLC) cells with TGF $\beta 1$ induced EMT to varying extents at molecular and/or functional levels. Intriguingly, the increase in number of CD133+ cells upon TGF $\beta 1$ treatment was highest for cell lines containing a significant number of hybrid E/M cells (identified as SLUG+ $\mathrm{CDH} 1+)$, indicating that the hybrid E/M population may be more 'poised' to give rise to CSCs [35]. 
Extensive heterogeneity in EMT has been observed in NSCLC cells [73]. H1975 and A549 cell lines have been reported to be predominantly hybrid $\mathrm{E} / \mathrm{M}[19,35,74,75]$ and plastic in their ability to move along the EMP spectrum $[55,76]$. However, whether these hybrid cell lines are more tumorigenic in vitro and in vivo as compared to their epithelial and mesenchymal counterparts still remains to be quantified. A recent study compared the tumor-initiation potential of morphologically distinct colonies obtained by A549, and observed that the subpopulation showing intermediate levels of E-cadherin and Vimentin had the maximum tumor-initiating capacity [77]. However, these experiments were done at a population level, thus it could not be identified whether this clone consisted of co-existing E-cadherin ( $\mathrm{CDH} 1+)$ and Vimentin $(\mathrm{VIM}+)$ cells or if individual cells expressed both $\mathrm{CDH} 1$ and VIM simultaneously. Recent computational methods can help dissect the system to differentiate between these two possibilities [78], and may enable mapping of EMP with stemness in NSCLC.

\section{Prostate and pancreatic cancer}

Various cell surface markers have been used to isolate CSCs in prostate cancer, including CD133 and CD44 ${ }^{\text {hi }} C D 24^{\text {lo }}[79]$. Similar to experiments in keratinocytes and for A549 lung cancer cells, PC3 cells were cultured to give rise to different clones with varying self-renewal potential. The clones containing the highest frequency of CSCs formed tightly packed colonies, indicating a more epithelial nature. They also expressed $\beta$-catenin and CD44, indicating a part "molecular EMT" [80]. Further analysis using PC3 subclones established that higher metastatic and tumor-initiating traits were limited to cells that did not exhibit a full-blown EMT. Further, knockdown of E-cadherin in these tumor-initiating cells enhanced invasiveness, but reduced their ability to form spheroids and colonize other organs in NOD/SCID mice [81]. Conversely, overexpression of E-cadherin in more mesenchymal PC-3 clones restricted invasiveness but increased the spheroid-formation capability [81]. These results are reminiscent of observations in breast cancer that knockdown of E-cadherin, which can induce a full-blown EMT, can restrict the metastatic potential of cells [82]. Reinforcing observations were recently reported in a mouse model of pancreatic cancer, where the "late hybrid" cells, but not the "most mesenchymal" cells were found to be metastatically advantageous with specific proliferative, metabolic, and signalling processes associated with them [9]. Therefore, in prostate and pancreatic cancer, "the more the EMT, the more the stemness" dogma does not hold true; instead, the 'stemness window' seems to lie on an epithelial or hybrid E/M range of values on the 'EMP axis'.

The dynamics of EMT/MET in prostate cancer is undergoing extensive investigation [20,83], and drivers of MET such as OVOL1/2 have been identified in prostate cancer cells [84]. Inducible models can help quantify the tumor-initiation potential as a function of EMT at varying time points, and single-cell analysis can help map the percentage of CSCs dynamically as cells undergo EMT/ MET [19]. Intriguingly, prostate cancer cells were shown to exhibit spontaneous switching among epithelial, mesenchymal and hybrid E/M subpopulations, with the hybrid ones being the most plastic [85]. Phenotypic stability factors such as GRHL2 were proposed to increase the relative stability of hybrid E/M cells $[11,13]$. Whether such plasticity of hybrid E/M cells is necessary or sufficient for them to self-renew and/or form tumors in vivo remain to be deciphered.

\section{Squamous cell carcinoma}

Recent findings in squamous cell carcinoma (SCC) have shed new light on EMP, and its association with stemness. Using a DMBA/TPA induced mouse model of cutaneous SCC, Pastushenko and colleagues demonstrated that FAT1 loss causes the appearance of a hybrid E/M 
cell state that co-expresses both epithelial and mesenchymal markers [86]. This state was associated with increased metastasis. This role for FAT1 deletion appeared to be specific for SCC, as FAT1 deletion in mouse lung tumours promoted the development of lung SCC over adenocarcinoma. Interestingly, in the same study, the authors also used a more mesenchymal tumour model driven by KRAS activation and p53 deletion targeted to the mouse hair follicle. In this model, FAT loss also caused appearance of a hybrid E/M cell state. Therefore, FAT1 loss appeared to induce a hybrid cell state from both epithelial and mesenchymal starting populations.

The authors elaborated on this mechanism by showing that FAT1 loss acts through two separate signalling pathways; CAMK2-CD44-SRC-YAP-ZEB1 activates a mesenchymal program, and CAMK2-EZH2-SOX2 activates an epithelial program [86]. Therefore, the hybrid state is induced through a balance of epithelial and mesenchymal signals. This agrees with studies cited in the preceding sections of this review, which have suggested that the hybrid cell state is governed by a balance of epithelial and mesenchymal transcriptional networks.

Contrastingly, another recent study has demonstrated the existence of a specific stem cell regulatory network alongside the EMP spectrum [87]. Here, using a cell line model of oral SCC and human tumour specimens, the authors identified a stem cell sub-population that could differentiate into both epithelial and mesenchymal lineages. This stem cell sub-population retained the epithelial marker EpCAM alongside a CD44 ${ }^{\text {hi }} \mathrm{CD} 24^{+}$stem cell marker profile and activation of mesenchymal transcriptional networks, and was predictive of metastatic status in human archival tumour specimens. Alongside its hybrid E/M signature, this stem cell sub-population activated a distinct transcriptional signature that was not shared with either its epithelial or mesenchymal counterparts, suggesting a specific stem cell regulatory network at work alongside EMP spectrum.

\section{A coherent model of EMP and stemness}

Put together, these observations suggest that hybrid E/M phenotypes are capable of forming tumors in vitro and in vivo, and may be the driving force behind metastatic dissemination. However, the following questions remain: a) If cells gain stemness during EMT, do they lose it during MET or is stemness maintained as a part of 'cellular memory'? b) Do any changes in molecular, morphological and/or functional traits of EMT directly contribute to stemness and/or plasticity? c) Is higher stemness seen in hybrid E/M states a consequence of the cells co-expressing both sets of markers, or is the 'un-differentiated' state regulated independently of EMP? In other words, is stemness dependent on the position of a cell within the EMP spectrum? Recent experiments decoding the dynamics of EMT induction in a time-dependent and/or dose-dependent manner in a cancer cell population may be helpful in answering some of these questions, particularly when combined with single cell analysis [18, 19, 88, 89].

We propose a coherent model that can resolve existing confusions around the association of EMT (or EMP) with stemness. As a first approximation, we consider that all phenotypes - epithelial, mesenchymal and hybrid E/M - have the potential to be stem-like; however, this potential is likely to be maximum for hybrid E/M cells. In representing this process (Fig 3), we have ignored the diversity of EMP trajectories and collapsed multi-dimensional EMP into one axis: the $x$-axis denotes the position of a cell along the EMP spectrum. The $y$-axis in this representation denotes the stemness-differentiation rheostat, similar to the ideas represented in Waddington's landscape, where valleys at the bottom denote terminally differentiated states [90]. Just as multi-dimensional EMP is collapsed onto the $x$-axis, the $y$-axis represents multi-dimensional stemness including both self-renewal potential and multilineage potential, which may in reality be semi-independent from each other [87]. The z-axis in this landscape signifies the potential (or stability) of a cell state. The 
deeper a given valley is, the more stable the corresponding state is. Thus, stem cells with similar coordinates on $x$ and $y$ axes can still have different $z$-axis coordinates (i.e. varying stability). A balance between stability and plasticity of various stem cell states can play an important role in propagating metastatic dissemination.

In this framework, cells en route through the transition in either direction ( $E$ to $M$ or vice-versa) usually become relatively more de-differentiated, which may contribute to the ability to self-renew as well as the ability to give rise to other cell types (multilineage potential). In other words, cells undergoing EMT/MET encounter a spectrum of de-differentiated states which may facilitate higher stemness. However, the association of hybrid E/M states and stemness is neither exclusive nor exhaustive. In this landscape populated by multiple states, switching to another state with a different value along the $x$-axis is not necessarily always accompanied by change along the $y$-axis too, or vice versa. For example, cells with an amoeboid phenotype have been associated with stemness traits and metastatic progression whilst having no epithelial features [91, 92]. Therefore, amoeboid cells might be an example of a phenotype that sits within the completely mesenchymal end of the spectrum but nevertheless exhibits heightened stemness [93].

This model allows many possible couplings between EMP and stemness, and highlights a higher likelihood of hybrid E/M cells existing in more 'stem-like' states. However, the level of interdependence between these axes is currently unknown, and is a topic of active investigation. Also unknown is the range of 'stemness' gene expression signatures. Specific gene expression signatures have been associated with the stemness axis in cancer [87, 94], but we do not yet know whether the stemness signature is universal across the entire EMP spectrum. Instead, the stemness signature may be contextual, based on position within the EMP spectrum. In order to answer this question, single cell approaches will be required that enable resolution of both transcriptional and functional attributes $[89,95]$ in order to identify all of the stem cell phenotypes existing across the EMP spectrum.

Stabilization of multiple cellular states (z-axis) within this framework may be achieved through mutually inhibitory feedback loops which may exhibit hysteresis or 'cellular memory'.. The more the self-stabilizing feedback loops, usually, the deeper the valley corresponding to that state is. Whether hybrid E/M states are stabilized by a balance of epithelial and mesenchymal 'teams' of players (similar to 'teams' seen in small cell lung cancer and melanoma [96, 97]), or there exist a bonafide 'team' of stabilizers of hybrid E/M phenotypes remains to be identified. In other words, it is possible that hybrid cell states are stabilized through molecules which may not be either EMTinducers or MET-inducers, but bona fide inducers for hybrid E/M phenotype(s). Recent work demonstrating the existence of a specific stem cell regulatory network alongside the EMP spectrum suggests that stem cell regulatory factors may contribute to multistability [87, 98], and indeed may represent a class of stabilization factors for hybrid E/M phenotype(s); for instance, SLUG has been implicated in stabilizing hybrid E/M state and promote stemness across multiple cancers [29, 99].

A limitation of our model is that the EMP status of a cell is represented by only one value whereas, as discussed earlier, EMP is a multi-dimensional process. For instance, cells en route through EMT can have coordinates along at least three additional semi-independent axes (not shown in the model): molecular EMT, morphological EMT and functional EMT. Similarly, multiple axes of stemness (multi-lineage potential, metabolic reprogramming, self-renewal etc.) are collapsed into one. Thus, we should exercise extreme caution while defining hybrid EMT state(s) to prevent its association with stemness from becoming an unfalsifiable hypothesis. Nevertheless, in reconciling recent findings, this model provides a coherent conceptual framework that points a way forward for the field to aid the interpretation of new findings, and clarify some of the most pressing unsolved questions. 


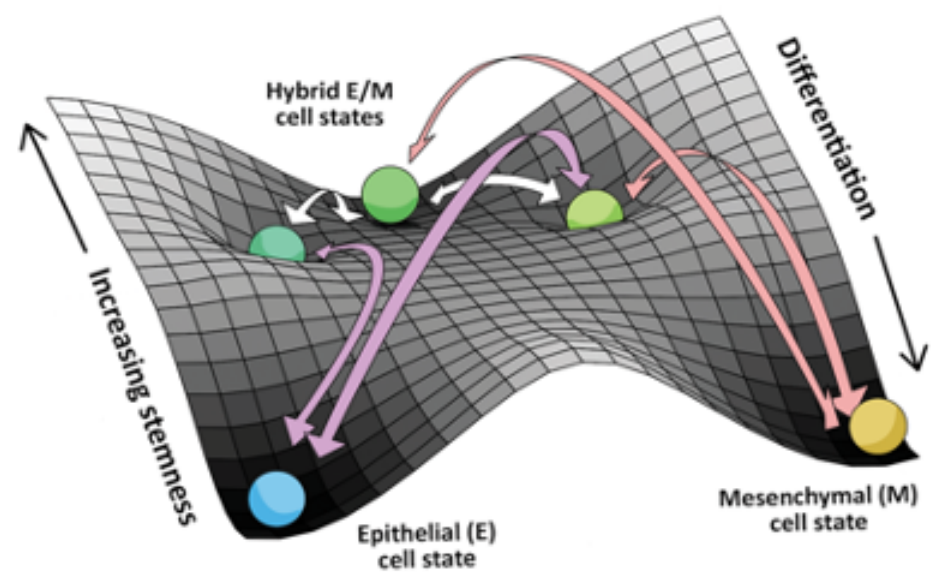

Figure 3: Adaptation of Waddington's landscape showing coupling between EMP (x-axis), stemness (y-axis), and stability of cell states (z-axis). EMP is plotted as a transdifferentiation process where epithelial and mesenchymal states are lower valleys (terminally differentiated states), whereas hybrid E/M states may be more 'pluripotent' (placed higher in the landscape, i.e. in a valley with a higher $y$-axis component than that of 'terminally differentiated' states). Arrows show different possible bidirectional transitions that are allowed in this landscape. 
Acknowledgements MKJ was supported by Ramanujan Fellowship (SB/S2/RJN-049/2018) awarded by Science and Engineering Research Board, Department of Science and Technology, Government of India; and by InfoSys Foundation, Bangalore. ASD acknowledges support from Prime Ministers' Research Fellowship (PMRF). AB is supported by Animal Free Research UK.

Author contributions MKJ and AB conceptualized and edited the review; SS and ASD prepared the first version and associated artwork.

Conflict of Interest The authors declare no conflict of interest.

\section{References:}

1. Gupta GP, Massagué J (2006) Cancer metastasis: building a framework. Cell 127:679-695

2. Celià-Terrassa T, Kang Y (2016) Distinctive properties of metastasis-initiating cells. Genes Dev 30:892-908. https://doi.org/10.1101/gad.277681.116

3. Celià-Terrassa T, Jolly MK (2020) Cancer Stem Cells and Epithelial-to-Mesenchymal Transition in Cancer Metastasis. Cold Spring Harb Perspect Med 10:a036905. https://doi.org/10.1101/cshperspect.a036905

4. Bocci F, Levine H, Onuchic JN, Jolly MK (2019) Deciphering the dynamics of epithelial-mesenchymal transition and cancer stem cells in tumor progression. Curr Stem Cell Reports 5:11-21

5. Jolly MK, Ware KE, Gilja S, et al (2017) EMT and MET: necessary or permissive for metastasis? Mol Oncol 11:755-769. https://doi.org/10.1002/1878-0261.12083

6. Pastushenko I, Blanpain C (2019) EMT Transition States during Tumor Progression and Metastasis. Trends Cell Biol 29:212-226.

https://doi.org/10.1016/j.tcb.2018.12.001

7. Lu M, Jolly MK, Levine $H$, et al (2013) MicroRNA-based regulation of epithelial-hybridmesenchymal fate determination. Proc Natl Acad Sci U S A 110:18174-9.

https://doi.org/10.1073/pnas.1318192110

8. Pastushenko I, Brisebarre A, Sifrim A, et al (2018) Identification of the tumour transition states occurring during EMT. Nature 556:463-468.

https://doi.org/10.1038/s41586-018-0040-3

9. Simeonov KP, Byrns CN, Clark ML, et al (2020) Single-cell lineage and transcriptome reconstruction of metastatic cancer reveals selection of aggressive hybrid EMT states. bioRxiv 245787. https://doi.org/10.1101/2020.08.11.245787

10. Biddle A, Liang X, Gammon L, et al (2011) Cancer stem cells in squamous cell carcinoma switch between two distinct phenotypes that are preferentially migratory or proliferative. Cancer Res 71:5317-5326. https://doi.org/10.1158/0008-5472.CAN-111059

11. Biswas K, Jolly M, Ghosh A (2019) Stability and mean residence times for hybrid epithelial/mesenchymal phenotype. Phys Biol 16:025003.

https://doi.org/10.1088/1478-3975/aaf7b7

12. Gupta PB, Fillmore CM, Jiang G, et al (2011) Stochastic state transitions give rise to 
phenotypic equilibrium in populations of cancer cells. Cell 146:633-644. https://doi.org/10.1016/j.cell.2011.07.026

13. Tripathi S, Chakraborty $P$, Levine $H$, Jolly MK (2020) A mechanism for epithelialmesenchymal heterogeneity in a population of cancer cells. PLoS Comput Biol 16:e1007619. https://doi.org/10.1101/592691

14. Katsuno Y, Meyer DS, Zhang Z, et al (2019) Chronic TGF- $\beta$ exposure drives stabilized EMT, tumor stemness, and cancer drug resistance with vulnerability to bitopic mTOR inhibition. Sci Signal 12:eaau8544.

https://doi.org/10.1126/scisignal.aau8544

15. Jia W, Deshmukh A, Mani SA, et al (2019) A possible role for epigenetic feedback regulation in the dynamics of the Epithelial-Mesenchymal Transition (EMT). Phys Biol 16:066004. https://doi.org/10.1101/651620

16. Jia W, Tripathi S, Chakraborty P, et al (2020) Epigenetic feedback and stochastic partitioning during cell division can drive resistance to EMT. Oncotarget 11:26112624. https://doi.org/10.18632/oncotarget.27651

17. Biddle A, Gammon L, Liang X, et al (2016) Phenotypic Plasticity Determines Cancer Stem Cell Therapeutic Resistance in Oral Squamous Cell Carcinoma. EBioMedicine 4:138-45. https://doi.org/10.1016/j.ebiom.2016.01.007

18. Celià-Terrassa T, Bastian C, Liu DD, et al (2018) Hysteresis control of epithelialmesenchymal transition dynamics conveys a distinct program with enhanced metastatic ability. Nat Commun 9:5005. https://doi.org/10.1038/s41467-018-07538-7

19. Karacosta LG, Anchang B, Ignatiadis N, et al (2019) Mapping Lung Cancer EpithelialMesenchymal Transition States and Trajectories with Single-Cell Resolution. Nat Commun 10:5587. https://doi.org/10.1101/570341

20. Stylianou N, Lehman ML, Wang C, et al (2019) A molecular portrait of epithelialmesenchymal plasticity in prostate cancer associated with clinical outcome. Oncogene 38:913-934. https://doi.org/10.1038/s41388-018-0488-5

21. Schmidt JM, Panzilius E, Bartsch HS, et al (2015) Stem-Cell-like Properties and Epithelial Plasticity Arise as Stable Traits after Transient Twist1 Activation. Cell Rep 10:131-139. https://doi.org/10.1016/j.celrep.2014.12.032

22. Cheung KJ, Ewald AJ (2014) Illuminating breast cancer invasion: diverse roles for cell-cell interactions. Curr Opin Cell Biol 30:99-111.

https://doi.org/10.1016/j.ceb.2014.07.003

23. Schaeffer D, Somarelli JA, Hanna G, et al (2014) Cellular migration and invasion uncoupled: increased migration is not an inexorable consequence of epithelial-tomesenchymal transition. Mol Cell Biol 34:3486-99.

https://doi.org/10.1128/MCB.00694-14

24. Devaraj V, Bose B (2019) Morphological State Transition Dynamics in EGF-Induced Epithelial to Mesenchymal Transition. J Clin Med 8:911. https://doi.org/10.3390/jcm8070911

25. Foroutan M, Bhuva DD, Lyu R, et al (2018) Single sample scoring of molecular phenotypes. BMC Bioinformatics 19:404. https://doi.org/10.1186/s12859-018-2435-4

26. Puram S V., Tirosh I, Parikh AS, et al (2017) Single-Cell Transcriptomic Analysis of Primary and Metastatic Tumor Ecosystems in Head and Neck Cancer. Cell 171:16111624. https://doi.org/10.1016/j.cell.2017.10.044 
27. Cheung KJ, Padmanaban V, Silvestri V, et al (2016) Polyclonal breast cancer metastases arise from collective dissemination of keratin 14-expressing tumor cell clusters. Proc Natl Acad Sci 113:E854-E863.

https://doi.org/10.1073/pnas.1508541113

28. Tripathi S, Jolly MK, Woodward WA, et al (2018) Analysis of hierarchical organization in gene expression networks reveals underlying principles of collective tumor cell dissemination and metastatic aggressiveness of inflammatory breast cancer. Front Oncol 8:244. https://doi.org/https://doi.org/10.1101/204388

29. Subbalakshmi AR, Sahoo S, Biswas K, Jolly MK (2021) A computational systems biology approach identifies SLUG as a mediator of partial Epithelial-Mesenchymal Transition (EMT). Cells Tissues Organs in press. https://doi.org/10.1159/000512520

30. Steinbichler TB, Dudas J, Ingruber J, et al (2020) Slug Is A Surrogate Marker of Epithelial to Mesenchymal Transition (EMT) in Head and Neck Cancer. J Clin Med 9:2061. https://doi.org/10.3390/jcm9072061

31. Leroy P, Mostov KE (2007) Slug Is Required for Cell Survival during Partial EpithelialMesenchymal Transition of HGF-induced tubulogenesis. J Cell Sci 18:1943-1952. https://doi.org/10.1091/mbc.E06

32. Schinke H, Pan M, Akyol M, et al (2020) Partial epithelial-to-mesenchymal transition is prognostic and associates with Slug in head and neck cancer. bioRxiv 346692

33. Karaosmanoğlu O, Banerjee S, Sivas H (2018) Identification of biomarkers associated with partial epithelial to mesenchymal transition in the secretome of slug overexpressing hepatocellular carcinoma cells. Cell Oncol 41:439-453. https://doi.org/10.1007/s13402-018-0384-6

34. Sterneck E, Poria DK, Balamurugan K (2020) Slug and E-Cadherin: Stealth Accomplices? Front Mol Biosci 7:138. https://doi.org/10.3389/fmolb.2020.00138

35. Andriani F, Bertolini G, Facchinetti F, et al (2016) Conversion to stem-cell state in response to microenvironmental cues is regulated by balance between epithelial and mesenchymal features in lung cancer cells. Mol Oncol 10:253-71.

https://doi.org/10.1016/j.molonc.2015.10.002

36. Mandal M, Ghosh B, Anura A, et al (2016) Modeling continuum of epithelial mesenchymal transition plasticity. Integr Biol 8:167-176.

https://doi.org/10.1039/C5IB00219B

37. Jolly MK, Tripathi SC, Jia D, et al (2016) Stability of the hybrid epithelial/mesenchymal phentoype. Oncotarget 7:27067-27084

38. Varankar SS, Kamble SS, Mali AM, et al (2020) Functional balance between TCF21Slug defines cellular plasticity and sub-classes in high-grade serous ovarian cancer. Carcinogenesis 41:515-526. https://doi.org/10.1093/carcin/bgz119

39. Aponte PM, Caicedo A (2017) Stemness in Cancer: Stem Cells, Cancer Stem Cells, and Their Microenvironment. Stem Cells Int 2017:5619472.

https://doi.org/10.1155/2017/5619472

40. Malta TM, Sokolov A, Gentles AJ, et al (2018) Machine Learning Identifies Stemness Features Associated with Oncogenic Dedifferentiation. Cell 173:338-354.e15. https://doi.org/10.1016/j.cell.2018.03.034

41. Grün D, Muraro MJ, Boisset JC, et al (2016) De Novo Prediction of Stem Cell Identity using Single-Cell Transcriptome Data. Cell Stem Cell 19:266-277.

https://doi.org/10.1016/j.stem.2016.05.010 
42. Gulati GS, Sikandar SS, Wesche DJ, et al (2020) Single-cell transcriptional diversity is a hallmark of developmental potential. Science 367:405-411.

https://doi.org/10.1126/science.aax0249

43. Efremov YR, Proskurina AS, Potter EA, et al (2018) Cancer stem cells: Emergent nature of tumor emergency. Front Genet 9:544.

https://doi.org/10.3389/fgene.2018.00544

44. Frisch SM, Schaller M, Cieply B (2013) Mechanisms that link the oncogenic epithelialmesenchymal transition to suppression of anoikis. J Cell Sci 126:21-29.

https://doi.org/10.1242/jcs.120907

45. Ho MM, Ng A V., Lam S, Hung JY (2007) Side population in human lung cancer cell lines and tumors is enriched with stem-like cancer cells. Cancer Res 67:4827-4833. https://doi.org/10.1158/0008-5472.CAN-06-3557

46. Shi Y, Fu X, Hua Y, et al (2012) The side population in human lung cancer cell line $\mathrm{NCl}-\mathrm{H} 460$ is enriched in stem-like cancer cells. PLoS One 7:e33358.

https://doi.org/10.1371/journal.pone.0033358

47. Ray A, Slama ZM, Morford RK, et al (2017) Enhanced Directional Migration of Cancer Stem Cells in 3D Aligned Collagen Matrices. Biophys J 112:1023-1036.

https://doi.org/10.1016/j.bpj.2017.01.007

48. Bertolini G, Roz L, Perego P, et al (2009) Highly tumorigenic lung cancer CD133+ cells display stem-like features and are spared by cisplatin treatment. Proc Natl Acad Sci U S A 106:16281-6. https://doi.org/10.1073/pnas.0905653106

49. Terris B, Cavard C, Perret C (2010) EpCAM, a new marker for cancer stem cells in hepatocellular carcinoma. J Hepatol 52:280-1.

https://doi.org/10.1016/j.jhep.2009.10.026

50. Liu S, Cong Y, Wang D, et al (2014) Breast cancer stem cells transition between epithelial and mesenchymal states reflective of their normal counterparts. Stem Cell Reports 2:78-91. https://doi.org/10.1016/j.stemcr.2013.11.009

51. Rasti A, Mehrazma M, Madjd Z, et al (2018) Co-expression of Cancer Stem Cell Markers OCT4 and NANOG Predicts Poor Prognosis in Renal Cell Carcinomas. Sci Rep 8:11739. https://doi.org/10.1038/s41598-018-30168-4

52. Takeda K, Mizushima T, Yokoyama Y, et al (2018) Sox2 is associated with cancer stem-like properties in colorectal cancer. Sci Rep 8:17639. https://doi.org/10.1038/s41598-018-36251-0

53. Yang L, Shi P, Zhao G, et al (2020) Targeting cancer stem cell pathways for cancer therapy. Signal Transduct Target Ther 5:8. https://doi.org/10.1038/s41392-020-0110-5

54. Neelakantan D, Zhou H, Oliphant MUJ, et al (2017) EMT cells increase breast cancer metastasis via paracrine GLI activation in neighbouring tumour cells. Nat Commun 8:15773. https://doi.org/10.1038/ncomms15773

55. Jolly MK, Preca B-T, Tripathi SC, et al (2018) Interconnected feedback loops among ESRP1, HAS2, and CD44 regulate epithelial-mesenchymal plasticity in cancer. APL Bioeng 2:031908. https://doi.org/10.1063/1.5024874

56. Iliopoulos D, Hirsch HA, Wang G, Struhl K (2011) Inducible formation of breast cancer stem cells and their dynamic equilibrium with non-stem cancer cells via IL6 secretion. Proc Natl Acad Sci 108:1397-1402. https://doi.org/10.1073/pnas.1018898108

57. Bocci F, Gearhart-Serna L, Boareto M, et al (2019) Toward understanding cancer 
stem cell heterogeneity in the tumor microenvironment. Proc Natl Acad Sci U S A 116:148-157. https://doi.org/10.1073/pnas.1815345116

58. Morel A-P, Lièvre M, Thomas C, et al (2008) Generation of breast cancer stem cells through epithelial-mesenchymal transition. PLoS One 3:e2888.

https://doi.org/10.1371/journal.pone.0002888

59. Mani SA, Guo W, Liao M-J, et al (2008) The epithelial-mesenchymal transition generates cells with properties of stem cells. Cell 133:704-715.

https://doi.org/10.1016/j.cell.2008.03.027

60. Scheel C, Weinberg RA (2012) Cancer stem cells and epithelial-mesenchymal transition: concepts and molecular links. Semin Cancer Biol 22:396-403.

https://doi.org/10.1016/j.semcancer.2012.04.001

61. Grosse-Wilde A, Fouquier d' Herouei A, Mclntosh E, et al (2015) Stemness of the hybrid epithelial/mesenchymal state in breast cancer and its association with poor survival. PLoS One 10:e0126522. https://doi.org/10.1371/journal.pone.0126522

62. Bierie B, Pierce SE, Kroeger C, et al (2017) Integrin- $\beta 4$ identifies cancer stem cellenriched populations of partially mesenchymal carcinoma cells. Proc Natl Acad Sci 114:E2337-2346. https://doi.org/10.1073/pnas.1618298114

63. Jolly MK, Huang B, Lu M, et al (2014) Towards elucidating the connection between epithelial-mesenchymal transitions and stemness. J R Soc Interface 11:20140962. https://doi.org/10.1098/rsif.2014.0962

64. Pastushenko I, Brisebarre A, Sifrim A, et al (2018) Identification of the tumour transition states occurring during EMT. Nature 556:463-468.

https://doi.org/10.1038/s41586-018-0040-3

65. Colacino JA, Azizi E, Brooks MD, et al (2018) Heterogeneity of human breast stem and progenitor cells as revelaed by transcriptional profiling. Stem Cell Reports 10:1596-1609. https://doi.org/10.1016/j.stemcr.2016.05.008

66. Al-Hajj M, Wicha MS, Benito-Hernandez A, et al (2003) Prospective identification of tumorigenic breast cancer cells. Proc Natl Acad Sci 100:3983-3988.

https://doi.org/10.1073/pnas.0530291100

67. Ginestier C, Hur MH, Charafe-Jauffret E, et al (2007) ALDH1 Is a Marker of Normal and Malignant Human Mammary Stem Cells and a Predictor of Poor Clinical Outcome. Cell Stem Cell 1:555-567. https://doi.org/10.1016/j.stem.2007.08.014

68. Goldman A, Majumder B, Dhawan A, et al (2015) Temporally sequenced anticancer drugs overcome adaptive resistance by targeting a vulnerable chemotherapy-induced phenotypic transition. Nat Commun 6:6139. https://doi.org/10.1038/ncomms7139

69. Kröger C, Afeyan A, Mraz J, et al (2019) Acquisition of a hybrid E/M state is essential for tumorigenicity of basal breast cancer cells. Proc Natl Acad Sci 116:7353-7362. https://doi.org/10.1073/pnas.1812876116

70. Shi R, Liu L, Wang F, et al (2020) Downregulation of cytokeratin 18 induces cellular partial EMT and stemness through increasing EpCAM expression in breast cancer. Cell Signal 76:109810. https://doi.org/10.1016/j.cellsig.2020.109810

71. Quan Q, Wang X, Lu C, et al (2020) Cancer stem-like cells with hybrid epithelial/mesenchymal phenotype leading the collective invasion. Cancer Sci 111:467-476. https://doi.org/10.1111/cas.14285

72. Eramo A, Lotti F, Sette G, et al (2008) Identification and expansion of the tumorigenic 
lung cancer stem cell population. Cell Death Differ 15:504-514. https://doi.org/10.1038/sj.cdd.4402283

73. Schliekelman MJ, Taguchi A, Zhu J, et al (2015) Molecular Portraits of Epithelial, Mesenchymal, and Hybrid States in Lung Adenocarcinoma and Their Relevance to Survival. Cancer Res 75:1789-1800. https://doi.org/10.1158/0008-5472.CAN-14-2535

74. Jolly MK, Tripathi SC, Jia D, et al (2016) Stability of the hybrid epithelial/mesenchymal phenotype. Oncotarget 7:27067-27084. https://doi.org/10.18632/oncotarget.8166

75. George JT, Jolly MK, Xu S, et al (2017) Survival Outcomes in Cancer Patients Predicted by a Partial EMT Gene Expression Scoring Metric. Cancer Res 77:64156428. https://doi.org/10.1158/0008-5472.CAN-16-3521

76. Wang $\mathrm{H}$, Zhang $\mathrm{H}$, Tang $\mathrm{L}$, et al (2013) Resveratrol inhibits TGF- $\beta 1$-induced epithelial-to-mesenchymal transition and suppresses lung cancer invasion and metastasis. Toxicology 303:139-46. https://doi.org/10.1016/j.tox.2012.09.017

77. Tièche CC, Gao Y, Bührer ED, et al (2018) Tumor Initiation Capacity and Therapy Resistance Are Differential Features of EMT-Related Subpopulations in the NSCLC Cell Line A549. Neoplasia 21:185-196. https://doi.org/10.1016/j.neo.2018.09.008

78. Jia D, George JT, Tripathi SC, et al (2019) Testing the gene expression classification of the EMT spectrum. Phys Biol 16:. https://doi.org/10.1088/1478-3975/aaf8d4

79. Moltzahn F, Thalmann GN (2013) Cancer stem cells in prostate cancer. Transl Androl Urol 2:242-253. https://doi.org/10.3978/j.issn.2223-4683.2013.09.06

80. Li H, Chen X, Calhoun-Davis T, et al (2008) PC3 human prostate carcinoma cell holoclones contain self-renewing tumor-initiating cells. Cancer Res 68:1820-5. https://doi.org/10.1158/0008-5472.CAN-07-5878

81. Celià-Terrassa T, Meca-Cortés Ó, Mateo F, et al (2012) Epithelial-mesenchymal transition can suppress major attributes of human epithelial tumor-initiating cells. J Clin Invest 122:1849-1868. https://doi.org/10.1172/JCI59218

82. Padmanaban V, Krol I, Suhail Y, et al (2019) E-cadherin is required for metastasis in multiple models of breast cancer. Nature 573:439-444.

https://doi.org/10.1038/s41586-019-1526-3

83. Cook DP, Vanderhyden BC (2020) Context specificity of the EMT transcriptional response. Nat Commun 11:2142. https://doi.org/10.1038/s41467-020-16066-2

84. Roca H, Hernandez J, Weidner S, et al (2013) Transcription Factors OVOL1 and OVOL2 Induce the Mesenchymal to Epithelial Transition in Human Cancer. PLoS One 8:e76773. https://doi.org/10.1371/journal.pone.0076773

85. Ruscetti M, Dadashian EL, Guo W, et al (2016) HDAC inhibition impedes epithelialmesenchymal plasticity and suppresses metastatic, castration-resistant prostate cancer. Oncogene 35:3781-95. https://doi.org/10.1038/onc.2015.444

86. Pastushenko I, Mauri F, Song Y, et al (2021) Fat1 deletion promotes hybrid EMT state, tumour stemness and metastasis. Nature 589:448-455. https://doi.org/10.1038/s41586-020-03046-1

87. Youssef G, Gammon L, Ambler L, et al (2020) Disseminating cells in human tumous acquire an EMT stem cell state that is predictive of metastasis. bioRxiv 029009. https://doi.org/10.1101/2020.04.07.029009

88. Jia W, Deshmukh A, Mani SA, et al (2019) A possible role for epigenetic feedback regulation in the dynamics of the epithelial-mesenchymal transition (EMT). Phys Biol 
16:. https://doi.org/10.1088/1478-3975/ab34df

89. McFaline-Figueroa JL, Hill AJ, Quu X, et al (2019) A pooled single-cell genetic screen identifies regulatory checkpoints in the continuum of the epithelial-to-mesenchymal transition. Nat Genet 51:1389-1398. https://doi.org/10.1038/s41588-019-0489-5

90. Jia D, Jolly MK, Kulkarni P, Levine H (2017) Phenotypic Plasticity and Cell Fate Decisions in Cancer : Insights from Dynamical Systems Theory. Cancers (Basel) 9:E70. https://doi.org/10.3390/cancers9070070

91. Cantelli G, Orgaz JL, Rodriguez-Hernandez I, et al (2015) TGF- $\beta$-Induced Transcription Sustains Amoeboid Melanoma Migration and Dissemination. Curr Biol 25:2899-2914. https://doi.org/10.1016/j.cub.2015.09.054

92. Rodriguez-Hernandez I, Maiques O, Kohlhammer L, et al (2020) WNT11-FZD7DAAM1 signalling supports tumour initiating abilities and melanoma amoeboid invasion. Nat Commun 11:5315. https://doi.org/10.1038/s41467-020-18951-2

93. Taddei ML, Giannoni E, Morandi A, et al (2014) Mesenchymal to amoeboid transition is associated with stem-like features of melanoma cells. Cell Commun Signal 12:24. https://doi.org/10.1186/1478-811X-12-24

94. Ben-Porath I, Thomson MWM, Carey VJVVJ, et al (2008) An embryonic stem cell-like gene expression signature in poorly differentiated aggressive human tumors. Nat Genet 40:499-507. https://doi.org/10.1038/ng.127

95. Wagner DE, Klein AM (2020) Lineage tracing meets single-cell omics: opportunities and challenges. Nat Rev Genet 21:410-427. https://doi.org/10.1038/s41576-0200223-2

96. Chauhan L, Ram U, Hari K, Jolly MK (2021) Topological signatures in regulatory network enable phenotypic heterogeneity in small cell lung cancer. Elife 10:e64522

97. Pillai M, Jolly MK (2021) Systems-level network modeling deciphers the master regulators of phenotypic plasticity and heterogeneity in melanoma. bioRxiv 434533. https://doi.org/10.1101/2021.03.11.434533

98. Pasani S, Sahoo S, Jolly MK (2021) Hybrid E/M phenotype(s) and stemness: a mechanistic connection embedded in network topology. J Clin Med 10:60. https://doi.org/10.1101/2020.10.18.341271

99. Lambert AW, Weinberg RA (2021) Linking EMT programmes to normal and neoplastic epithelial stem cells. Nat Rev Cancer. https://doi.org/10.1038/s41568-02100332-6 